\title{
Aroma and Bitter Extracts from South African Hops: Characterisation and Extraction Process Development using Supercritical $\mathrm{CO}_{2}$
}

\author{
Archery V, Madzimbamuto T and Ojumu T
}

\begin{abstract}
Supercritical fluid extraction (SFE) has become the preferred processing method over solvent extraction, due to its non-toxicity and its GRAS status (generally regarded as safe). An application is the extraction of aroma and bitter components of hops, in order to standardize it during use. South African hops are rather unique among worldwide cultivars, due to its origin's unique geography and terroir. This work explores the SFE using South African hops, characterizes the products and compares it with those obtained from worldwide suppliers. A process model of the extraction process is developed using literature solubility data and an equilibrium flash calculation to determine the selectivities. It is simulated using Aspen Batch Process Developer, and validated using pilot plant data. The RK-Aspen equation of state is used to model the vapour liquid equilibria of the volatile components. The technical feasibilities and economic viabilities are compared based on energy consumption.
\end{abstract}

Keywords - Supercritical fluid extraction, South African hops, process model, pilot plant

\section{INTRODUCTION}

Hops (Humulus lupulus L.) are cone structured plants that are cultivated in temperate areas. It forms part of the Cannabinaccea family that originates in China [1]. The male and female hops grow separately where the female hop produces the lupulin (resin) and the male crop is used to hybridise and create new varieties [2]. The hop plant contains flavours, resinous compounds and essential oil [3]. Hops are grouped into bitter, aroma and dual purpose hops. The bitter hops have high levels of acids (8-10\%). The aroma hops are used for craft beers as it has various flavours. Dual hops are suitable for both bittering and aroma [2]. Hops or their extracts are an important ingredient for the brewing of beer. The purpose of the extract in the final product of brewing was traditional to preserve the beer. In hop extracts, there are roughly two groups: flavour and bitter compounds. Both the flavour and the bitter taste have become the hallmark of western beers, ales and lagers. The cone of the flower is the source of flavour and the bitter compounds, as well as those compounds with preservation properties [4]. The bitter taste is regarded as a balance to the sweetness of the malts, while the flavour enhances its drinkability. Hops are grown where the climate consists of freezing winters and very long summer days. However, in George, South Africa, which lies $34^{\circ}$ south

Archery V, Madzimbamuto T and Ojumu T, Department of Chemical Engineering, Cape Peninsula University of Technology, P.O. Box 1906, Bellville, 7535, South Africa. of the equator hops flourish. Although George's summer days are three hours shorter than that of the northern hemisphere, South Africa has been successful in growing hops [5].

The first hops were introduced in South Africa in the 1650s. It was in 1652; where Jan van Riebeeck planted the first hop garden, which brewed its first beer two years later. A few years thereafter, the famous Capetonian wine was produced. Cape-Town was then known as "The Tavern of the Seas" as it as the stopover on the Dutch East India Route. South Africa would mainly import hops. However, this changed during the First World War. The South African hop industry developed in the 1970s when a local strain, Southern Brewer, was cultivated. This strain was adapted to the growing conditions in George, which led to the commercial production of hops. Over the years, more varieties were cultivated and today the industry thrives [5]. Some local varieties include African Queen; Southern Aroma, Southern Passion and Southern Star [6].

Hop cones can be processed into various forms such as hop-extracts, pellets or powder. According to Kupski 2017, the advantages of using hop extracts instead of hop pellets or powder is that it offers a control for the bitterness between various batches. This is due to a homogenous extract being produced. The production of hop extracts has advantages such as improved hop utilization, better stability when storing and reduction in transportation and storage cost [1]. These extracts are obtained either via organic solvent extract or supercritical fluid extraction. However, supercritical fluid extraction is preferred when processing food products, as there is no residue found in the final product. Solvent extraction leaves traces of the solvent used whereas supercritical carbon dioxide extraction is a non-toxic solvent. Solvent extraction yields a high amount of essential oil at lower cost. Traditionally, solvents such as hexane, ethanol, methanol or methylene chloride were used. However, only ethanol and liquid $\mathrm{CO}_{2}$ can be used nowadays, as they meet the requirements for generally acceptable solvent for food grade products (GRAS) [7]. To perform the ethanol extraction, the hop is mixed with an ethanol/water solution in an extractor. The ethanol travels through the hop bed and becomes enriched with hop components. The hop leaves are then called spent material. The polar hop material and ethanol mixture is known as "miscella". This mixture is pumped to the evaporation stage where the ethanol is removed via vacuum evaporation. The drawback with extraction using ethanol is that it is a wide spectrum solvent. It is thus not possible to fractionate more precisely the composition of the extract [7]. With supercritical carbon 
dioxide extraction, the process occurs under very high pressure. The plant material is placed in a stainless steel vessel and carbon dioxide is injected into the vessel. As the pressure builds in the vessel, the carbon dioxide becomes a solvent that extracts the desired components from the plant material. As the vessel is depressurised the carbon dioxide coverts to a gas again. This ensures that there is residue in the extract. This method produces extracts that are fresher, cleaner and a better aroma in comparison to that of steam distillation. This method is more desired as it uses low temperatures [8]. By simply adjusting the solvent power by manipulating the density through adjustment of temperature or pressure, it is possible to select the desired component.

The development of a process model allows for parameter prediction as it is possible to predict the behaviour of the extraction process. When modelling the extraction kinetics a few models are appropriate such as broken/intact cells models and shrinking core [1]. The shrinking core model assumes that the pores of the plant material is filled with extract and the solvent penetrates to the centre of the spherical material [9]. The broken/intact cell model divides the extract into broken cells (free oil) and into intact cells (tied oil) [1].

In literature for the hop-extraction by supercritical fluid extraction, Del Valle et al, produced hop-extract from Chilean hops. The investigation indicated that little increase in extraction occurred when operating at a pressure $>200$ bar. Thermal degradation increased when the temperature was $>40^{\circ} \mathrm{C}$ [10]. Kupski et al, extracted from hop pellets, where the highest yield was $7.1 \%$ at 200 bar and $55^{\circ} \mathrm{C}$. The optimal conditions were used to develop a mathematic model [1]. Hoshino et al, fractionated hop-extract ethanol solutions in a counter-current extraction column. The conditions which gave maximum column productivity were at $333.2 \mathrm{~K}$ and $8 \mathrm{MPa}$ with an $\mathrm{S} / \mathrm{F}=8.6$ [3]. Formato et al, investigated the SFE of $\alpha$ and $\beta$ acids. It was compared to cyclically pressurized solid-liquid extraction. Ethanol was used as a co-solvent [4]. Due to the novelty of hops, growing in South Africa, it has created the desire to explore the hop strain, in terms of their aroma and bitter characteristics. This exploration will enable the development of processes to produce extracts, and thus to enhance the value of this crop. A cleaner and safer method of extracting the desired components as well as providing a platform to create new flavours of beer can be developed. By characterising the constituents of the South African hop, other fields such as the pharmaceutical industry can benefit as this plant has been recognised for its medicinal activity for centuries. In this study, the focus is on extracting the hop extract and further separating it into its different components. This serves the purpose of characterisation of the hop.

This work is aimed at the extraction of hop pellets with and without a co-solvent at various pressures and temperatures. The second part of this work investigates the fractionation of hops from a solid matrix to separate the volatile compounds from the aroma compounds.

\section{EXPERIMENTAL METHOD}

\section{A. Materials and chemicals}

Carbon dioxide (99.9\%) was purchased from Air Liquide (Epping). Ethanol (99.9\%) was purchased from Sigma-Aldrich.

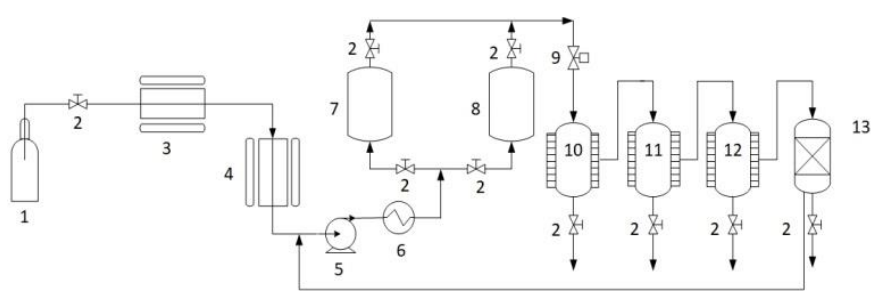

Fig. 1. Pilot plant for high pressure extraction with $\mathrm{CO}_{2}$ as a solvent. 1. $\mathrm{CO}_{2}$ cylinder, 2. Manual valve, 3. Chiller, 4. Cooler, 5. $\mathrm{CO}_{2}$ pump, 6. Heater, 7. Extractor I, 8. Extractor II, 9. Automated valve, 10. Separator I, 11. Separator II, 12. Separator III, 13. Filter.

\section{B. SEPAREX-SFE 5 pilot plant and procedure}

The supercritical $\mathrm{CO}_{2}$ extraction pilot plan is shown in fig 1. To determine the extraction operating condition for hops a thermodynamic model will be developed on Aspen. This model was created using vapour-liquid equilibrium data and equations of state. Once the operating conditions were obtained the extraction using the pilot plant was done. The feed was prepared by grinding the pellets and using a Tyler mesh sieve. The initial mass was recorded. The thermal equipment of the pilot plant was set. The $\mathrm{CO}_{2}$ was cooled and pumped; it is then heated by $6 . \mathrm{CO}_{2}$ flow rate is quantified by a flow meter. The $\mathrm{CO}_{2}$ was heated to the operating conditions for extraction $\left(40^{\circ} \mathrm{C}\right)$. The hops were loaded into 7 and 8 . The extraction baskets are stainless steel with a volume of $5 \mathrm{~L}$. The extractor baskets has porous disc at both ends of the baskets. Static extraction proceeded for 30 minutes. Once the valve was opened dynamic extraction begins. The $\mathrm{CO}_{2}$ became enriched with hop components. It then travelled to 10,11 and 12 where the $\mathrm{CO}_{2}$ drops the extract. The $\mathrm{CO}_{2}$ returned to its gaseous state. The $\mathrm{CO}_{2}$ is recycled into the process. The extract is sampled every 30 minutes. The co-solvent was then introduced where the polyphenols were extracted. For the fractionation from a solid matrix the same procedure was followed but a series of pressures are used, starting at the lowest pressure ( $150 \mathrm{bar}$ ). The pressure is then increase in 50 bar increments until 250 bar was reached. These samples were stored in a refrigerator. The samples were then sent for analysis. The extraction kinetics was modelled using mathematical models and the commercial simulator (Aspen Batch).

\section{Analysis of hop-extracts}

The types of analysis that will be used includes High Performance Liquid Chromatography (HPLC), Gas Chromatography Mass Spectrometer (GC-MS), Fourier Transform Infrared Resonance (FTIR) and Nuclear Magnetic Resonance (NMR).

\section{RESULTS AND DISCUSSION}

Preliminary experiments were conducted at a temperature of $40{ }^{\circ} \mathrm{C}$ and pressure of 300 bar. This resulted in a yield of $23.3 \%$. 


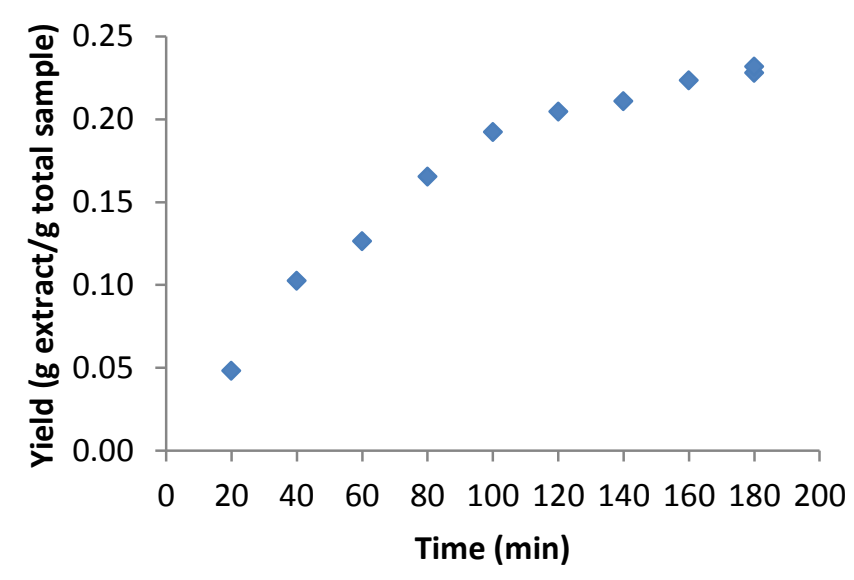

\section{CONCLUSION}

Based on preliminary experiments and the limited data produced the extraction from South African hops is a feasible process. Further experiments will indicate how temperature, pressure and $\mathrm{CO}_{2}$ flow rate affects the extraction rate. The effect of a co-solvent (ethanol) will be investigated. The fractionation from a solid matrix will be conducted using various pressures.

\section{REFERENCES}

[1] S. C. Kupski, E. J. Klein, E. A. da Silva, F. Palú, R. Guirardello, and M. G. A. Vieira, "Mathematical modeling of supercritical CO2extraction of hops (Humulus lupulus L.)," J. Supercrit. Fluids, vol. 130, no. March, pp. 347-356, 2017.

https://doi.org/10.1016/j.supflu.2017.06.011

[2] K. Dodds, "Hops - a guide for new growers," 2017.

[3] Y. Hoshino, M. Ota, Y. Sato, R. L. Smith, and H. Inomata, "Fractionation of hops-extract-ethanol solutions using dense $\mathrm{CO} 2$ with a counter-current extraction column," J. Supercrit. Fluids, vol. 136, no. December 2017, pp. 37-43, 2018.

https://doi.org/10.1016/j.supflu.2018.02.005

[4] A. Formato, M. Gallo, D. Ianniello, D. Montesano, and D. Naviglio, "Supercritical fluid extraction of $\alpha$ - And $\beta$-acids from hops compared to cyclically pressurized solid-liquid extraction," J. Supercrit. Fluids, vol. 84, pp. 113-120, 2013. https://doi.org/10.1016/j.supflu.2013.09.021

[5] S. Brewer, "OUR DREAM IS TO BRING PEOPLE TOGETHER Meet some locals," pp. 1-3, 2019.

[6] S. A. Hops, "7/29/2019 SOUTH AFRICAN HOPS - ZAHOPS.com," pp. 2018-2020, 2019.

[7] C. W. Bamforth, "The processing of hops," Brew. New Technol., pp. 123148, 2006. https://doi.org/10.1533/9781845691738.123

[8] B. Oil, "Pre-Feasibility Study Essential Oils Distillation Unit," Office, no. $15,2010$.

[9] P. H. Sovova, "Life Long Learning Intensive Course " Process Intensification by High Pressure Technologies - Actual Strategies for Energy and Resources Conservation " Modelling of Efficiency and Energy Consumption of SCF Extraction from Plant Materials Modelling of Efficiency and Energy Consumption of SCF Extraction from Plant Materials," 2014.

[10] J. M. Del Valle, O. Rivera, O. Teuber, and M. T. Palma, "Supercritical $\mathrm{CO} 2$ extraction of Chilean hop (Humulus lupulus) ecotypes," J. Sci. Food Agric., vol. 83, no. 13, pp. 1349-1356, 2003. https://doi.org/10.1002/jsfa.1547 